\title{
Tribological characteristics of self-assembled nanometer film deposited on phosphorylated 3-aminopropyltriethoxysilane
}

\author{
$\mathbf{J} \mathbf{L I}$ and $\mathbf{X ~ Z ~} \mathbf{L} \mathbf{I}^{\dagger, *}$ \\ School of Mechanical and Automation Engineering, Shanghai Institute of Technology, Shanghai 200235, \\ P.R. China \\ ${ }^{\dagger}$ English group, Shouguang Experimental School, Shandong, Shouguang 261041, P.R. China
}

MS received 12 September 2008

\begin{abstract}
Thin films deposited on the phosphonate 3-aminopropyltriethoxysilane (APTES) self-assembled monolayer (SAM) were prepared on the hydroxylated silicon substrate by a self-assembling process from specially formulated solution. Chemical compositions of the films and chemical state of the elements were detected by X-ray photoelectron spectrometry (XPS). The thickness of the films was determined with an ellipsometer, while the morphologies and nanotribological properties of the samples were analyzed by means of atomic force microscopy (AFM). As the results, the target film was obtained and reaction might have taken place between the thin films and the silicon substrate. It was also found that the thin films showed the lowest friction and adhesion followed by APTESSAM and phosphorylated APTES-SAM, whereas silicon substrate showed high friction and adhesion. Microscale scratch/wear studies clearly showed that thin films were much more scratch/wear-resistant than the other samples. The superior friction reduction and scratch/wear resistance of thin films may be attributed to low work of adhesion of nonpolar terminal groups and the strong bonding strength between the films and the substrate.
\end{abstract}

Keywords. Thin films; friction; adhesion; scratch/wear.

\section{Introduction}

Microelectromechanical systems (MEMS) and emerging nanoelectromechanical systems (NEMS) are expected to have a major impact on our lives, much like the way that the integrated circuit has affected information technology (Anonymous 1977; Roukes 2001). However, due to the large surface area to volume to volume ratio in MEMS/NEMS devices as the size scale shrinks, currently many potential applications for MEMS/NEMS are not really practical. Many studies have revealed the profound negative influence of stiction, friction and wear on the efficiency, power output, and steady-state speed of micro/nanodynamic devices (Bhushan 1998; Bhushian 1999; Kayali et al 1999; Arney 2001). The self-assembled monolayer (SAM) has gained growing interest over the past years because it has advantageous characteristics of well-defined structure, strong head group-substrate binding and dense packing of hydrocarbon chains. Indeed SAM considerably reduces friction and adhesion and is found useful in various microelectromechanical systems (MEMS) devices (Depalma and Tillman 1989; Bhushan et al 1995; Tsukruk et al 1996; Boshui et al 1998; Dugger et al 2000).

A number of studies have been done on the nanotribological properties of different SAMs (Cha and Kim

\footnotetext{
*Author for correspondence (lishuyang2003.student@ sina.com)
}

2001; Bhushan 2005), but the study of the rare earth films on the nanotribological behaviour is still lacking. In this study, thin films deposited on the phosphorylated 3-aminopropyltriethoxysilane (APTES) self-assembled monolayer (SAM) were prepared on the silicon substrates. Different means, such as X-ray photoelectron spectroscopy (XPS), atomic force microscopy (AFM), ellipsometer and contact angle measurements, were applied to investigate the structure and nanotribological properties of the prepared films.

\section{Experimental}

\subsection{Preparation of thin films on the phosphorylated APTES-SAM}

APTES was purchased from Aldrich Chemical Company, Inc. A single-crystal silicon wafer polished on one side was used as substrate for the SAM transfer. Other reagents were of analytical grade. Deionized water was used throughout the experiment. For the preparation of the target solution, APTES, toluene, ethanol, acetonitrile, phosphorus oxychloride, and collidine were commercially obtained and used without further purification. The silicon substrates were cleaned with 'piranha' solution $\left(\mathrm{H}_{2} \mathrm{SO}_{4}: \mathrm{H}_{2} \mathrm{O}_{2}=7: 3(\mathrm{v}: \mathrm{v})\right)$ (caution: this solution reacts violently with organics), then exposed to a solution of APTES in toluene $(2 \% \mathrm{~V}: \mathrm{V}, 24 \mathrm{~h}$, 


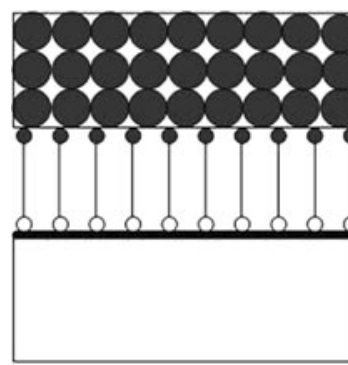

thin films

Phosphorylated APTES-SAM

silicon substrate

Figure 1. Schematic of growth of thin films.

room temperature), followed by $\mathrm{POCl}_{3}$ in acetonitrile $(0 \cdot 2 \mathrm{M}$ $\mathrm{POCl}_{3}, 0 \cdot 2 \mathrm{M}$ collidine, $20 \mathrm{~min}$, room temperature), according to the described procedure (Katz et al 1991; Buscher et al 1996). This treatment resulted in surface richness in phosphonate groups $\left(-\mathrm{PO}(\mathrm{OH})_{2}\right)$, which adsorbed a layer of thin films when immersed in pre-prepared solution, then naturally cooled in a desiccator. Schematic of growth of thin films on phosphorylated APTES-SAM is shown in figure 1 .

\subsection{Description of apparatus and test procedures}

Chemical compositions of the films and chemical state of the elements were analyzed by a PHI-5702 XPS system, using $\mathrm{Mg}-\mathrm{K} \alpha$ radiation operating at $250 \mathrm{~W}$ and pass energy of $29.35 \mathrm{eV}$. The binding energy of $\mathrm{C} 1 \mathrm{~s}(284.6 \mathrm{eV})$ was used as the reference. The resolution for the measurement of the binding energy is about $\pm 0 \cdot 3 \mathrm{eV}$. The static contact angles were measured in ambient air (relative humidity $40 \%$ ) using an OCA-20 contact angle measurement device (DataPhysics Instruments Gmbh). Distilled water was used as the spreading reagent. Measurements were done at least on three samples, and were made at a minimum of three different spots on each sample. The contact angles were typically reproducible to within $\pm 2^{\circ}$. The thickness of the films was measured on an ellipsometer (V-VASE with AutoRetarder from J.A. Woollam Co., polarizer-retarder-sample-rotating analyzer configuration) which was equipped with a He-Ne laser $(632.8 \mathrm{~nm})$ set at an incident angle of $70^{\circ}$. The index of refraction for the refraction was taken to be 1.45 . Thickness data was obtained by averaging five measurements at different spots of each sample surface. The thickness was recorded to an accuracy of $\pm 0 \cdot 3 \mathrm{~nm}$.

The surface morphology and the nanotribological properties of the prepared films were investigated using an SPM9500 atomic force microscope (NanoScope IIIa) produced by Shimadzu Corporation (Kyoto, Japan). Square pyramidal $\mathrm{Si}_{3} \mathrm{~N}_{4}$ tips with a nominal $50 \mathrm{~nm}$ radius mounted on goldcoated triangular $\mathrm{Si}_{3} \mathrm{~N}_{4}$ cantilevers with spring constants of $0.6 \mathrm{~N} / \mathrm{m}$ were used. The adhesion and friction data were measured ten times at each interesting location and average data values were obtained. Adhesive forces were measured using the so called 'force calibration plot'. Friction forces were measured according to the procedures given by Binnig et al (1986). By following the friction force calibration procedures developed by Glosli and Gary McClelland (1993), voltages corresponding to friction forces can be converted to force units. Coefficient of friction is obtained from the slope of friction force data measured as a function of normal loads.

For the scratch and wear tests, specially fabricated microtips were used. These microtips, consisted of singlecrystal natural diamond, ground to the shape of a three-sided pyramid, with an apex angle of $80^{\circ}$ and tip radius of about $50 \mathrm{~nm}$, were mounted on a platinum-coated stainless steel cantilever beam whose stiffness was $50 \mathrm{~N} / \mathrm{m}$. Samples were scanned orthogonal to the long axis of the cantilever with loads ranging from 20 to $100 \mu \mathrm{N}$ to generate scratch/wear marks. Observations of the sample surface before and after the wear tests were done by scanning parallel to the long axis of the cantilever with loads ranging from 0.5 to $1 \mu \mathrm{N}$. The parallel scans enabled near-zero wear of the sample surface and also eliminated post-data analysis errors in surface feature height. Scratch/wear tests were performed over a scan area of $2 \times 2 \mu \mathrm{m}$ at a scan rate of $10 \mathrm{~Hz}$. The reported scratch/wear depths are an average of six runs at separate instances. In this study, all the measurements were carried out in ambient conditions $\left(22^{\circ} \mathrm{C}, \mathrm{RH} 40-44 \%\right)$.

\section{Results and discussion}

\subsection{Characterization of the prepared films}

Figure 2 shows a series of AFM images taken over regions $1.0 \times 1.0 \mu \mathrm{m}$ of specimens at various stages of the film deposition process, where panel (a) refers to the bare cleaned silicon substrate, (b) APTES-SAM on the silicon substrates, (c) the phosphorylated APTES-SAM, and (d) to the as-deposited thin films on the phosphorylated APTES-SAM. It can be seen that the surface of the silicon substrates (figure 2(a)) is clean and smooth with surface root-mean-square (rms) roughness in the range of $0 \cdot 2-0 \cdot 3 \mathrm{~nm}$. Figure 2 (b) is uniform and homogenous with surface rms roughness about $0.522 \mathrm{~nm}$. The phosphorylated APTES-SAM (figure 2(c)) becomes more and more uniform and homogeneous with rms roughness about $0.393 \mathrm{~nm}$. The possible reason is that the size of $-\mathrm{PO}(\mathrm{OH})_{2}$ groups is bigger than the terminal groups $-\mathrm{NH}_{2}$ which leads to the $-\mathrm{PO}(\mathrm{OH})_{2}$ terminal molecules provided a more densely packed arrangement than the $-\mathrm{NH}_{2}$ terminal molecules. After the deposition of the thin films (figure 2(d)) on the phosphorylated APTES-SAM, many differences are visible in the corresponding AFM images, viz. the surface of the as-deposited films is rough and quite densely round-looking particles with the rms roughness to be about $0.863 \mathrm{~nm}$, which shows wide potential application in lubrication and wear protection.

The XPS spectra applied to detect the chemical states of some typical elements for the prepared films are shown in 

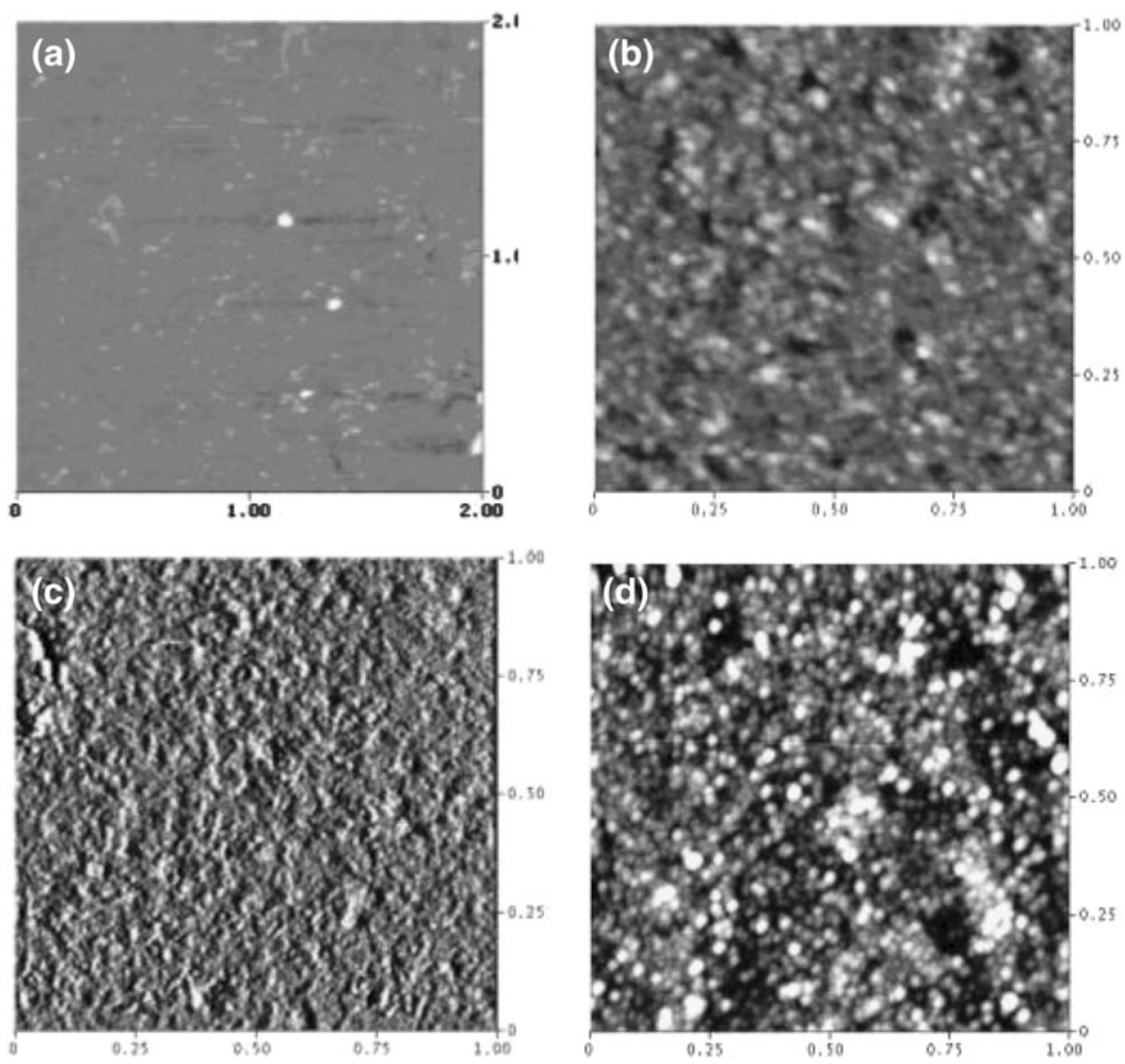

Figure 2. AFM images of (a) bare silicon substrate, (b) APTES-SAM, (c) phosphorylated APTESSAM and (d) thin films.

figures 3-6. XPS survey and single scan spectra of APTESSAM (figure 3) shows contributions from the substrate and the film are: silicon $(15.5 \%)$, carbon $(56.3 \%)$, oxygen $(20.2 \%)$, and nitrogen $(8.0 \%)$. Nitrogen is detected indicating successful APTES-SAM deposition, since this element is contained only in this film material. Figure 4 shows singlescan XPS spectra of $\mathrm{N} 1 \mathrm{~s}$ peak decomposed into two different nitrogen species occurring in different binding states. The peak at $400.8 \mathrm{eV}$ is assigned to the protonated aliphatic amino groups, while that at $399.5 \mathrm{eV}$ may be ascribed to the aliphatic amino groups. This interpretation is consistent with the one given by Bierbaum et al (1995).

After in situ phosphorylation of the APTES-SAM, the $P 2_{\mathrm{p}}$ at $134.5 \mathrm{eV}$ is observed (figure 5), which is assigned to the $\mathrm{P}$-atoms in $-\mathrm{PO}(\mathrm{OH})_{2}$ group and $\mathrm{N}$ signal is absent. It indicates that the terminal $-\mathrm{NH}_{2}$ group in the APTES-SAM has been phosphorylated and transformed to $-\mathrm{PO}(\mathrm{OH})_{2}$ group successfully and completely.

The contact angles of distilled water on silicon substrate and the prepared films were measured using a contact angle measurement. The APTES-SAM has a contact angle of $50 \pm$ $2^{\circ}$, which is consistent with a moderately polar surface where

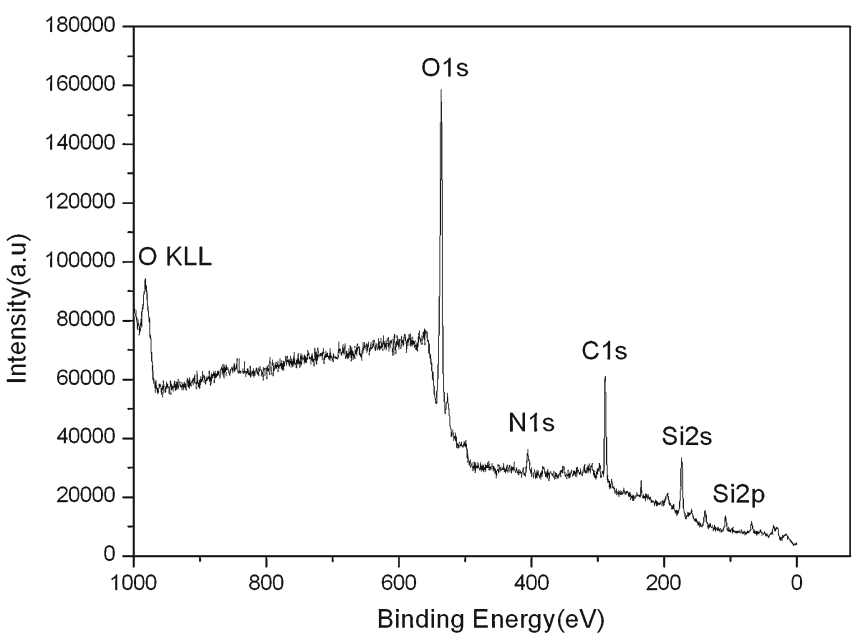

Figure 3. XPS survey and single scan spectra of APTES film.

the amino group is oriented upward. After phosphorylated in situ for $20 \mathrm{~min}$, a contact angle of $24 \pm 2^{\circ}$ is recorded for the phosphorylated surface. The contact angle of thin films 


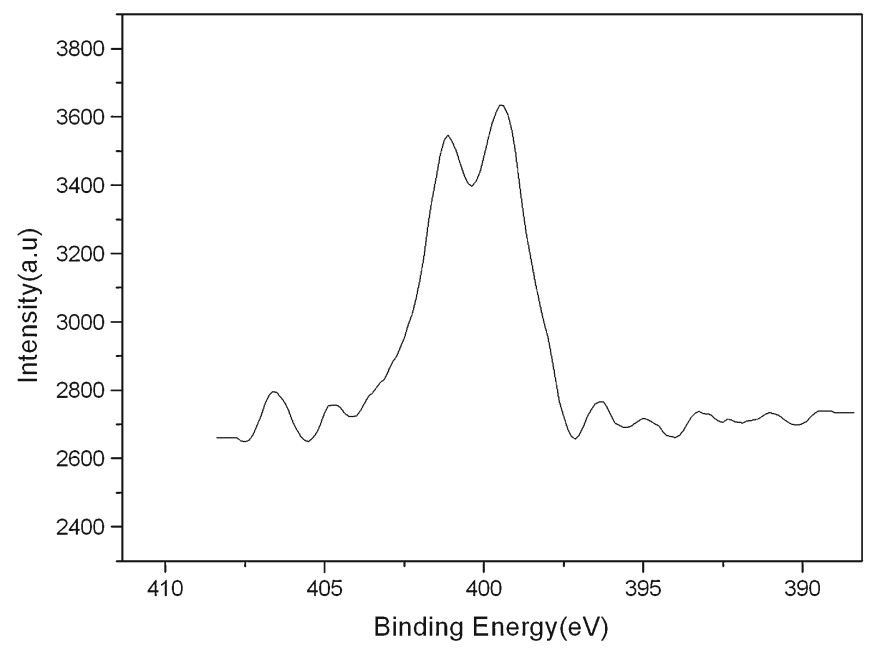

Figure 4. XPS spectrum of the N $1 \mathrm{~s}$ region of an APTMS film.

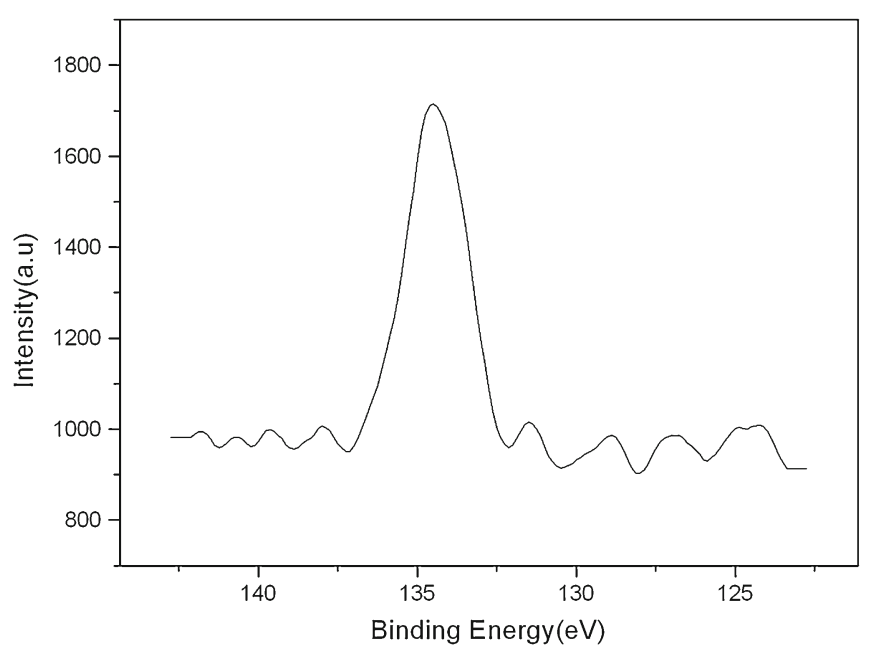

Figure 5. XPS spectrum of the $P 2_{\mathrm{p}}$ region in APTMS film.

deposited on the phosphorylated APTES-SAM increases to $66 \pm 2^{\circ}$.

In our work, the thickness of the prepared films on glass substrates is determined with ellipsometer. The averaged thickness of the APTES-SAM is about $7.5 \mathrm{~nm}$, which matches with the projection of a normally extended molecular chain on the surface. The thickness is hardly changed after the $-\mathrm{NH}_{2}$ is phosphorylated to $-\mathrm{PO}(\mathrm{OH})_{2}$ group, which indicates that a monolayer of phosphorylated APTES has been prepared on glass substrates. The thickness increases by about $15 \mathrm{~nm}$ after the phosphorylated APTES-SAM is immersed in solution, which also shows that thin films have been successfully obtained.

\subsection{Nanotribological properties of prepared films}

3.2a Adhesion, friction and work of adhesion: Figure 6 shows the average values of the adhesion force and

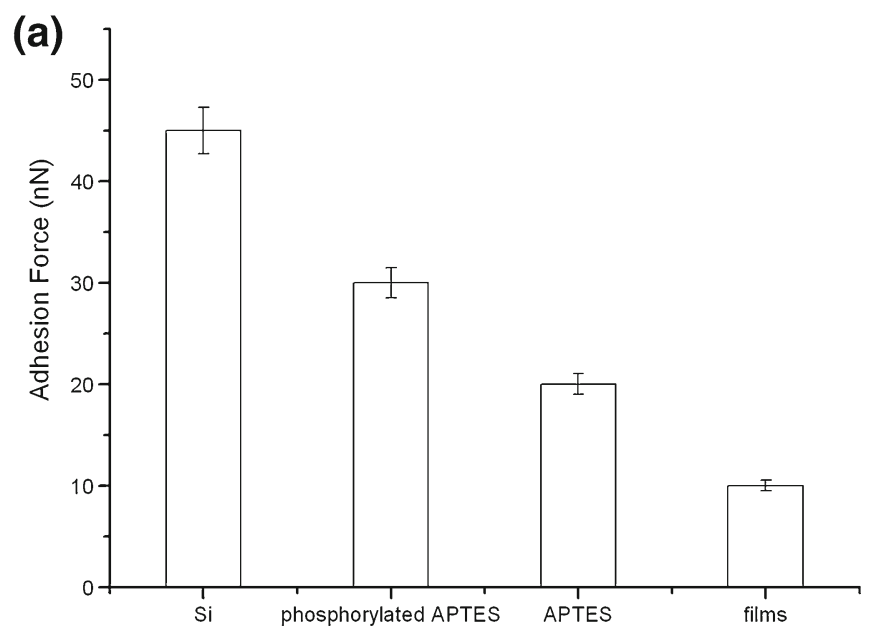

(b)

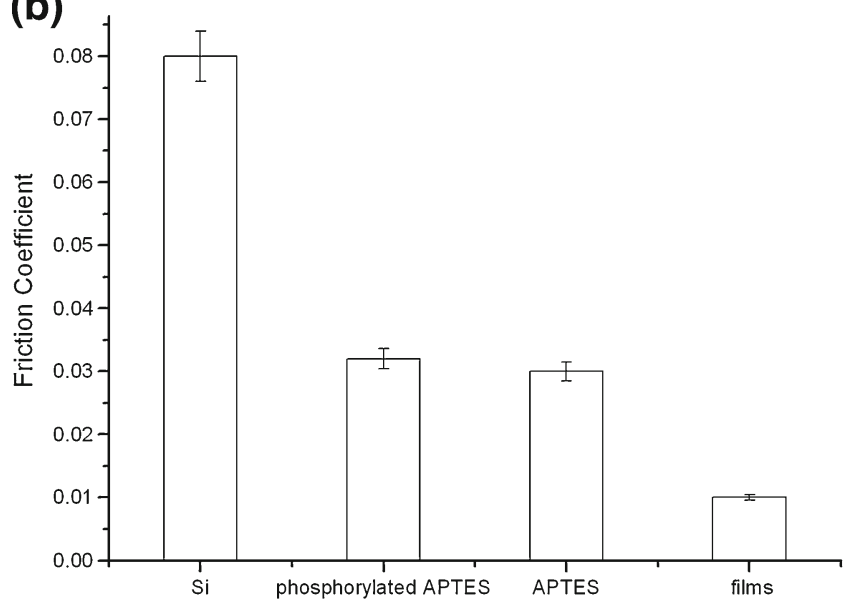

Figure 6. (a) Adhesive forces of silicon and prepared film and (b) coefficients of friction of silicon and films.

coefficients of friction of four kinds of flat surfaces measured by contact mode AFM under an applied normal load of $20 \mathrm{nN}$ and a scan rate of $10 \mathrm{~Hz}$. It shows that SAMs can reduce the adhesive and frictional forces of silicon substrate. In particular, thin films exhibit the lowest adhesive force and coefficient of friction. It means that the prepared films can be used as effective molecular lubricants for micro/nanodevices fabricated from silicon. Based on the data, the ranking of adhesive forces $F_{\mathrm{a}}$ is in the following order: $F_{\mathrm{a}-\mathrm{Si}}>$ $F_{\text {a-phosphorylated APTES }}>F_{\mathrm{a} \text {-APTE }}>F_{\mathrm{a} \text {-films. }}$. And the ranking of the coefficients of friction is in the following order: $\mu_{\mathrm{Si}}>\mu_{\text {phosphorylated APTES }} \approx \mu_{\mathrm{APTES}}>\mu_{\text {films }}$.

Based on Young-Dupre equation, work of adhesion $W_{\mathrm{a}}$ (the effort required to pull apart the unit area of the solid interface (Israelachvili 1985)) was summarized:

$$
W_{\mathrm{a}}=\gamma_{1 \mathrm{a}}\left(1+\cos \theta_{1}\right),
$$

where $\gamma_{1 \mathrm{a}}$ is the surface tension of liquid-air interface and $\theta_{1}$ is the water contact angle of liquid and flat. From figures 6 and 7 , it is clear that the adhesive force and friction coefficient decreases as the work of adhesion decreases. It implies 


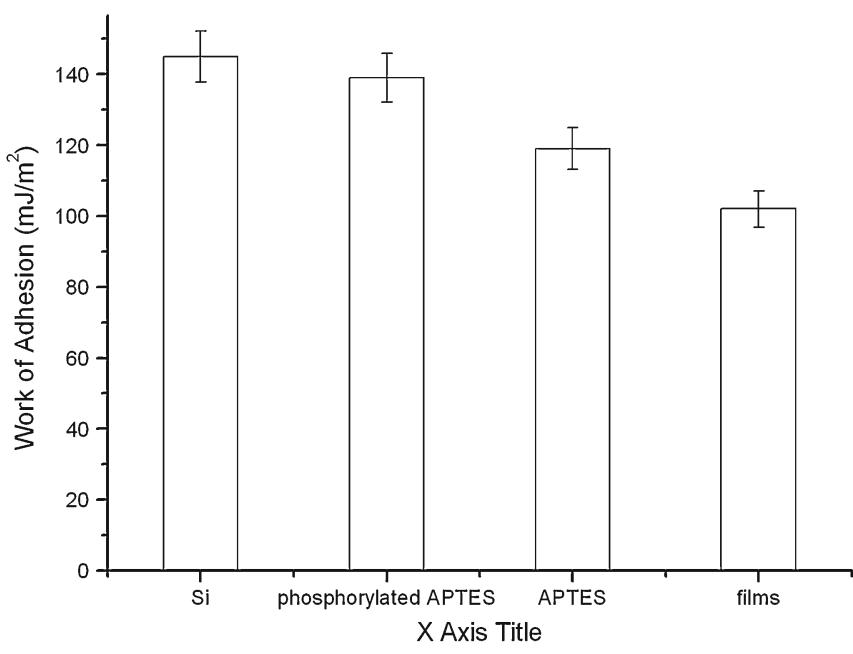

Figure 7. Work of adhesion of friction of silicon and films.

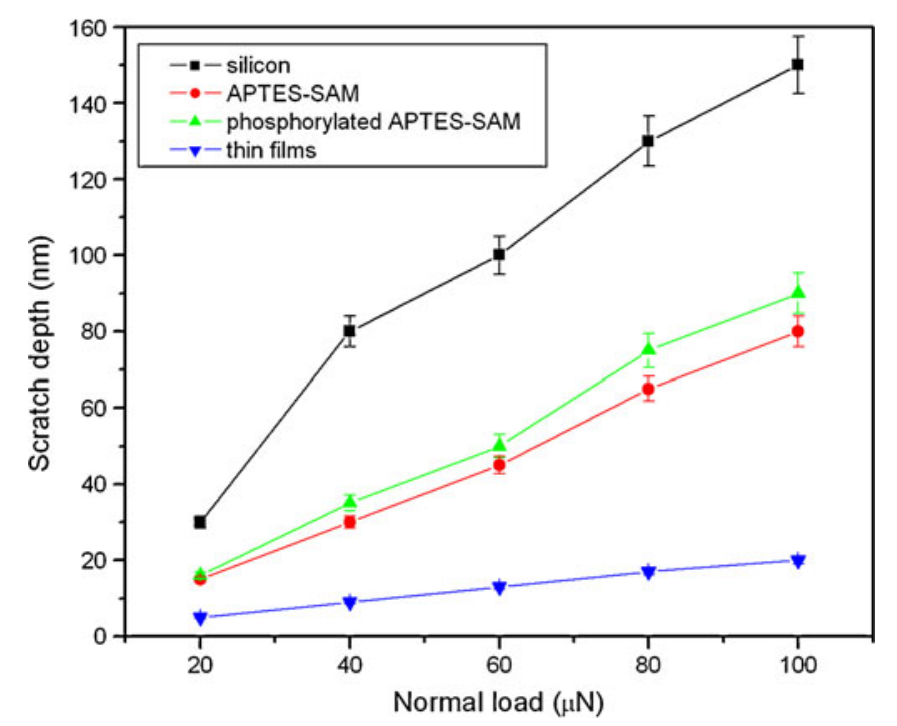

Figure 8. Scratch depths for 10 cycles as a function of load.

that the capillary force acting between the tip and the flat surfaces affected seriously on nano-adhesion and nano-friction. From figure 6, it can be also found that APTES-SAM and phosphorylated APTES-SAM have polar surface groups $\left(-\mathrm{NH}_{2}\right.$ and $-\mathrm{PO}(\mathrm{OH})_{2}$ groups), thus lead to larger $W_{\mathrm{a}}$ and eventually larger adhesive forces. The films do not have polar surface groups, thus have a smaller $W_{\mathrm{a}}$ and adhesive force than APTES-SAM and phosphorylated APTES-SAM.

3.2b Scratch/wear tests: As explained earlier, the scratch tests of silicon and the prepared films were studied by making scratches for 10 cycles with varying loads. Figure 8 shows a plot of scratch depth vs. normal load for various samples. Scratch depth increases with increasing normal load. APTES-SAM and phosphorylated APTES-SAM show similar scratch resistance. From the data, it is clear that the thin films show the best scratch resistance compared with the silicon substrate, APTES-SAM and phosphorylated APTESSAM. The increase in scratch depth with normal load is very small and all depths are less than $20 \mathrm{~nm}$, while the silicon substrate and APTES-SAM finally reach depths of about $80 \mathrm{~nm}$ and $150 \mathrm{~nm}$, respectively.

Wear tests were conducted on the samples by wearing the same region for 30 cycles at a normal load of $20 \mu \mathrm{N}$, while observing wear depths at different intervals $(1,5,15$, 20 and 30 cycles). This would give information as to the progression of wear of the films. The wear depths observed are plotted against the number of cycles in figure 9. For all the samples, the wear depth increases almost linearly with increasing number of cycles. This suggests that material is removed layer by layer in all the materials. Here also, thin films exhibit lesser increase in wear depth (slope) than the other samples. APTES-SAM wears less than the silicon substrate, which shows similar wear characteristics to the phosphorylated APTES-SAM. Combined with the scratch/wear data and the thickness of the films, it can be found that the scratch/wear depth of APTES-SAM and phosphorylated APTES-SAM are much more than their thickness $(7.5 \mathrm{~nm})$. On the contrary, the scratch/wear depth of thin films does not exceed its thickness $(15 \mathrm{~nm})$ all the time. It shows that APTES-SAM and phosphorylated APTES-SAM are seriously destroyed with the increase of normal load and number of cycles, while thin films are still not completely worn at last. The scratch/wear results all indicate that thin films have better surface mechanical properties than silicon substrate, APTES-SAM or phosphorylated APTES-SAM.

The $-\mathrm{PO}(\mathrm{OH})_{2}$ group on the APTES-SAM provides net negative charge, thus promoting the process of self-assembly. Then $-\mathrm{PO}(\mathrm{OH})_{2}$ can provide a $\mathrm{P}=\mathrm{O}$ bond as complexing group to react after adsorption, thus improving the bonding strength between the films and the silicon substrates. In

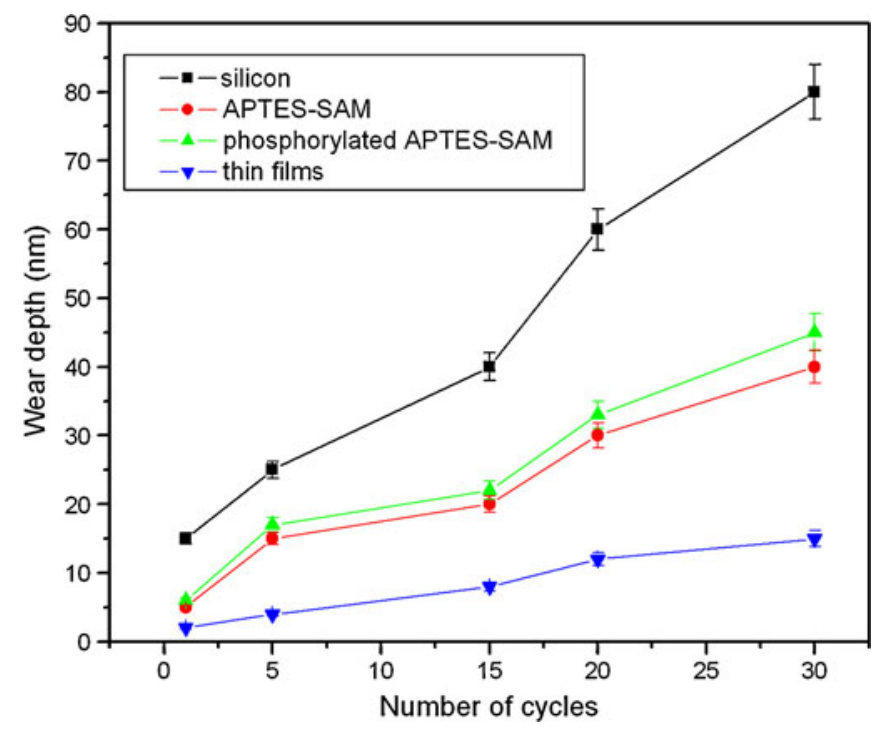

Figure 9. Wear depths as a function of number of cycles. 
addition, Hertzian cone cracks can occur when the normal stress exceeds a critical value as the AFM tip slides over the surface. Friction forces during sliding reduce this critical value. In short, strong bonding strength between the films and the silicon substrate and low coefficient of friction are responsible for the superior scratch/wear resistance of thin films.

\section{Conclusions}

In this work, the nanotribological properties of silicon substrate, APTES-SAM, phosphorylated APTES-SAM, and the thin films were characterized by an AFM. The thin films showed the lowest friction and adhesion followed by APTES-SAM and phosphorylated APTES-SAM, while silicon substrate showed high friction and adhesion. Microscale scratch/wear studies clearly showed that thin films were much more scratch/wear resistant than the other samples. The superior friction reduction and scratch/wear resistance of thin films may be attributed to low work of adhesion of non-polar terminal groups and the strong bonding strength between the films and the substrate.

It is thus concluded that the prepared thin films could be used for protection from scratching as well as reducing friction.

\section{Acknowledgement}

The authors would like to thank help from Shanghai Institute of Technology, Project Number: YJ2009-14.

\section{References}

Anonymous 1977 Microelectromechanical systems: Advanced materials and fabrication methods, NMAB-483 (Washington DC: National Academy Press)

Arney S 2001 MRS Bull. 26296

Bhushan B 1998 Tribology issues and opportunities in Mems (Dordrecht: Kluwer Academic)

Bhushan B 2005 Wear 2591507

Bhushan B, Israelachvili J N and Landmann U 1995 Nature 374 607

Bhushian B 1999 Handbook of micro/nanotribology (Boca Raton, FL: CRC) 2nd ed

Bierbaum K, Kinzler M, Wöll Ch, Gruzne M, Hähner G, Heid S and Effenberger F 1995 Langmuir 11512

Binnig G, Quate C F and Gerber Ch 1986 Phys. Rev. Lett. 56930

Boshui C, Yi Y and Junxiu D 1998 J. Chin. Rare Earth Soc. 16220

Buscher T C, McBranch D and DeQuan L 1996 J. Am. Soc. 118 2950

Cha K H and Kim D E 2001 Wear 2511169

Depalma V and Tillman N 1989 Langmuir 5868

Dugger M T, Senft D C and Nelso G C 2000 ACS Symp. Ser. 741 455

Glosli J N and Gary McClelland M 1993 Phys. Rev. Lett. 701960

Israelachvili J N 1985 Intermolecular and surface forces (New York: Academic Press)

Katz H E, Scheller G, Putvinski T M, Schilling M L, Wilson W L and Chidsey C E D 1991 Science 2541485

Kayali S, Lawton R, Smith B H and Irwin L W 1999 EEE Links 5 10

Roukes M 2001 Physics World $\mathbf{1 4} 25$

Tsukruk V V, Bliznyuk V N, Hazel J, Visser D and Everson M P 1996 Langmuir 124840 